\title{
The Structure of Aggrecan Fragments in Human Synovial Fluid

\author{
Evidence for the Involvement in Osteoarthritis of a Novel Proteinase Which Cleaves the Glu 373-Ala 374 \\ Bond of the Interglobular Domain
}

\begin{abstract}
John D. Sandy, ${ }^{\star *}$ Carl R. Flannery, * Peter J. Neame, ${ }^{\star *}$ and L. Stefan Lohmander
*Shriners Hospital for Crippled Children, Tampa Unit, Tampa, Florida 33612; ${ }^{\ddagger}$ Department of Biochemistry and Molecular Biology, School of Medicine, University of South Florida, Tampa, Florida 33612; and ${ }^{\S}$ Department of Orthopedics, University Hospital, S-22185
\end{abstract}

Lund, Sweden

\begin{abstract}
Synovial fluid was collected from patients with recent knee injury and from patients with early or late stage osteoarthritis. Chondroitin sulfate-substituted aggrecan fragments present in these fluids, and in normal bovine synovial fluid, were purified by cesium chloride gradient centrifugation, enzymically deglycosylated and fractionated by gel filtration on Superose-12. Each sample contained two major aggrecan core protein populations with apparent molecular masses of $\sim 90 \mathrm{kD}$ and $150 \mathrm{kD}$. For all samples, $\mathbf{N H}_{2}$-terminal analysis of both populations gave a single major sequence beginning ARGSV. This $\mathbf{N H}_{2}$ terminus results from cleavage of the human aggrecan core protein at the Glu 373-Ala 374 bond within the interglobular domain between the $G 1$ and $G 2$ domains. Cleavage at this site also occurs during control and interleukin-1 stimulated aggrecan catabolism in bovine cartilage explant cultures (Sandy, J., P. Neame, R. Boynton, and C. Flannery. 1991. J. Biol. Chem. 266:8683-8685).

These results indicate that the major aggrecan fragments present in both osteoarthritic human synovial fluid and in normal bovine synovial fluid are large, being composed of a short $\mathbf{N H}_{2}$-terminal stretch of the interglobular domain, the $\mathbf{G 2}$ domain, the keratan sulfate domain, and variable lengths of the chondroitin sulfate domain(s). We conclude that the release of aggrecan fragments from articular cartilage into the synovial fluid seen at all stages of human osteoarthritis (Lohmander, $L$. S. 1991. Acta Orthop. Scand. 62:623-632) is promoted by the action of a normal cartilage proteinase which cleaves the Glu 373-Ala 374 bond of the interglobular domain. (J. Clin. Invest. 1992. 89:1512-1516.) Key words: articular cartilage • proteoglycan $\bullet$ core protein $\bullet$ cartilage proteinase $\bullet \mathrm{NH}_{2}$-terminal sequence
\end{abstract}

\section{Introduction}

The disease mechanisms active in osteoarthritis (OA) ${ }^{1}$ are unclear, but changes in the biochemical and biomechanical properties of joint cartilage, changes in chondrocyte matrix synthe-

Address correspondence to Dr. J. D. Sandy, Shriners Hospital for Crippled Children, Tampa Unit, Tampa, FL 33612.

Received for publication 15 October 1991 and in revised form 8 January 1992.

1. Abbreviations used in this paper: CS, chondroitin sulfate; OA, osteoarthritis.

J. Clin. Invest.

(c) The American Society for Clinical Investigation, Inc.

$0021-9738 / 92 / 05 / 1512 / 05 \$ 2.00$

Volume 89, May 1992, 1512-1516 sis, and, finally, a gradual destruction of the matrix are characteristic of the disease process. This sequence of events has been best documented by the use of animal models of posttraumatic OA (1-3). Cartilage proteoglycan fragments are released to joint fluid after knee injury and in early stages of both posttraumatic and primary OA in the human (4-6). An important role for matrix metalloproteinases in both normal turnover of connective tissue matrix and in the tissue destruction seen in, for example, OA has been suggested $(7,8)$, and an imbalance between tissue metalloproteinases and inhibitors has been demonstrated in animal model OA cartilage (9), in joint fluids from patients with recent joint injury, and in OA joint fluids $(10,11)$. Further, increased expression of $\mathrm{mRNAs}$ for collagenase, stromelysin, and metalloproteinase inhibitor has been shown in synovial cells stimulated with interleukin- 1 and in synovial cells from rheumatoid or osteoarthritic joints (12-15). However, no definitive evidence has yet been presented which demonstrates that these enzymes are directly involved in cartilage matrix breakdown in OA. A detailed characterization of matrix fragments released from joint cartilage after trauma and in OA of the human could help identify the degradative mechanisms.

Characterization of aggrecan fragments released from bovine explants treated with interleukin-1 in vitro has demonstrated a major cleavage site within the interglobular domain of the proteoglycan core protein which releases large aggrecan fragments from the tissue (16). In the present investigation, we have isolated aggrecan fragments from joint fluids obtained from patients with recent knee joint injury or early or late stage knee OA. We show that the major aggrecan products present in these synovial fluids are relatively large and are composed of a segment of the interglobular domain attached to the G2 domain, the $\mathrm{KS}$ domain, and variable lengths of the chondroitin sulfate domain(s). (Aggrecan structural domains are defined in refs. 17 and 18.) The results are consistent with an accelerated loss of aggrecan from articular cartilage at all stages of human osteoarthritis (19), this loss being promoted by the action of a cartilage proteinase which cleaves human aggrecan at the Glu 373-Ala 374 bond.

\section{Methods}

Materials. Superose-12 (HR 10/30) and fast desalting (HR 10/10) columns were from Pharmacia LKB Biotechnology Inc., Piscataway, NJ. Chondroitinase $\mathrm{ABC}$ (protease free) from Proteus vulgaris and keratanase from Pseudomonas species IFO-13309 were from ICN Immunobiologicals, Lisle, IL. Keratanase II from Bacillus species KS 36 was from Seikagaku America Inc., Rockville, MD. All other reagents were analytical grade from commercial sources.

Synovial fluids. Human synovial fluid was collected from the knee joints of seven patients in connection with diagnostic or therapeutic arthrocentesis. Fluids were centrifuged at $3,000 \mathrm{~g}$ to remove cells and particulate material and then frozen at $-70^{\circ} \mathrm{C}$. The diagnosis was 
made through arthroscopy and radiological and clinical examination and details are given in Table I. Bovine synovial fluid was combined from the normal metacarpalphalangeal joints of nine steers.

Isolation of aggrecan fragments from joint fluid. Synovial fluid samples were adjusted to $4 \mathrm{M}$ guanidine- $\mathrm{HCl}, 0.05 \mathrm{M}$ sodium acetate, 0.1 mM PMSF, $5 \mathrm{mM}$ EDTA, $5 \mathrm{mM}$ iodoacetamide, $1 \mu \mathrm{g} / \mathrm{ml}$ pepstatin, pH 6.8, and centrifuged at $20,000 \mathrm{~g}$ for $30 \mathrm{~min}$ to remove any precipitate. Solid $\mathrm{CsCl}$ was then added to a density of $1.45 \mathrm{~g} / \mathrm{ml}$ and gradients established by centrifugation at $40,000 \mathrm{rpm}$ for $48 \mathrm{~h}$ at $10^{\circ} \mathrm{C}$. The tubes were sliced in three equal parts to yield fractions D1 (bottom) to D3, and samples were dialyzed at $4^{\circ} \mathrm{C}$ against $0.05 \mathrm{M}$ sodium acetate $\mathrm{pH}$ 6.8 with protease inhibitors (see above). After a final dialysis against water, the samples were lyophilized and portions were assayed for total protein or papain digested for assay of glycosaminoglycan. The insoluble gel collected from the top of the gradient tubes was dialyzed and lyophilized together with the top gradient fraction.

Assay and sequencing procedures. Proteoglycan and glycosaminoglycan were determined as chondroitin sulfate (CS) equivalents by dimethylmethylene blue (20), using shark chondroitin sulfate as standard. Protein was determined using the bicinchoninic acid assay kit from Pierce Chemical Co., Rockford, IL, with bovine serum albumin as standard. Amino acid sequence analysis was performed on a sequencer (473A or 477A; Applied Biosystems, Inc., Foster City, CA) with on-line phenylthiohydantoin analysis. For deglycosylation, D1 samples in $50 \mathrm{mM}$ Tris acetate, $10 \mathrm{mM}$ EDTA, pH 7.6, were treated at $37^{\circ} \mathrm{C}$ with chondroitinase $\mathrm{ABC}(0.075 \mathrm{U} / \mathrm{mg} \mathrm{CS})$ for $2 \mathrm{~h}$. Samples were adjusted to $5 \mathrm{mM}$ PMSF and $10 \mathrm{mM} N$-ethylmaleimide and incubated for $1 \mathrm{~h}$ with keratanase $(0.1 \mathrm{U} / \mathrm{mg}$ starting $\mathrm{CS})$ and finally with keratanase II $(0.0025 \mathrm{U} / \mathrm{mg}$ starting CS) for a further $1 \mathrm{~h}$. This procedure resulted in a $>90 \%$ reduction in the reactivity of all samples with dimethylmethylene blue. Core protein preparations from Superose- 12 were concentrated to $0.5 \mathrm{ml}$ and desalted on a fast desalting column eluted with water before drying for sequence analysis.

\section{Results}

Isolation of aggrecan fragments from synovial fluid. Synovial fluids from seven patients (see Table I for clinical data) were subjected to $\mathrm{CsCl}$ gradient centrifugation and the gradient fractions were assayed for glycosaminoglycan and protein. In all samples, $>94 \%$ of the glycosaminoglycan and $<2.5 \%$ of the total protein was recovered from the gradient in the bottom fraction $(D 1$, density $\sim 1.55 \mathrm{~g} / \mathrm{ml})$. This indicates that the bulk of the aggrecan fragments present in these synovial fluid samples were of high buoyant density and therefore rich in chondroitin sulfate relative to protein.

A portion of the D1 fractions from patients A and $F$ (Table I) were analyzed on Sepharose CL-2B in the presence of 5\% (wt/wt) hyaluronan and 4\% (wt/wt) link protein (not shown). The two profiles were very similar and showed the presence of a polydisperse population of CS-bearing fragments (peak Kav $\sim 0.55$ ) which did not form aggregates with hyaluronan and link protein. The fragments eluted later on Sepharose CL-2B than aggrecan monomer from mature human cartilage (peak Kav 0.38), but not as late as a tryptic digest of monomer (peak Kav 0.70) or a papain digest of monomer (peak Kav $\sim 0.79$ ). The results indicate that aggrecan fragments from these joint fluids completely lack a functional G1 domain and also that they have been cleaved, although not extensively, within the CS-bearing domains.

Consistent with these findings, fractionation of synovial fluid D1 aggrecan fragments from patients A through $G$ (Table I) on Superose-12 showed that in all cases the CS-bearing species were large enough to be excluded from Superose-12 (see Fig. 1, upper panels, for examples), which has an exclusion limit for dextrans of $\sim 300 \mathrm{kD}$. In addition, fractionation of deglycosylated D1 samples on Superose-12 (see Fig. 1, lower panels, for examples) indicated that the major aggrecan core protein species in all samples were in the apparent molecular mass range of $90-150 \mathrm{kD}$.

Characterization of core proteins. A detailed analysis of the $D 1$ aggrecan fragments from the three patients $A, D$, and $F$ was carried out (see Fig. 1, $a, b$, and $c$, respectively). Superose-12 profiles are shown for the D1 samples (Fig. 1, upper panels) and an equivalent portion of the corresponding deglycosylated samples (Fig. 1, lower panels). It can be seen that the D1 samples were contaminated to a varying degree with nonaggrecan protein, shown as $214 \mathrm{~nm}$ absorbance, which eluted in the included fractions with an apparent size below $66 \mathrm{kD}$. This material was presumably "trapped" with the D1 aggrecan during ultracentrifugation, possibly as a result of a high concentration of hyaluronan and protein in these samples. The identity of these proteins was not further investigated.

Table I. Clinical Data for Patient Samples

\begin{tabular}{|c|c|c|c|c|c|c|}
\hline Sample & $\begin{array}{l}\text { Age, } \\
\text { sex }\end{array}$ & Diagnosis* & Radiology $y^{*}$ & Cartilage at arthroscopy $y^{8}$ & Timell & Vol' $^{\prime}$ \\
\hline & & & & & & $m l$ \\
\hline A & $45 \mathrm{~F}$ & $\mathrm{ACL}$ & Normal & Chondral lesion & $3 d$ & 20 \\
\hline B & $27 \mathrm{M}$ & $\mathrm{ACL}+\mathrm{MM}$ & Normal & Clefts, softening & $2 w k$ & 10 \\
\hline $\mathrm{C}$ & $38 \mathrm{M}$ & $\mathrm{ACL}+\mathbf{M M}+\mathbf{L M}$ & Normal & Superficial fibrillations & $5 \mathrm{yr}$ & 20 \\
\hline D & $50 \mathrm{M}$ & $\begin{array}{l}\text { Medial } \\
\text { meniscectomy }\end{array}$ & $\begin{array}{l}\text { Osteophytes, minor decrease } \\
\text { of medial joint space }\end{array}$ & No data & $15 \mathrm{yr}$ & 20 \\
\hline $\mathrm{E}$ & $64 \mathrm{~F}$ & Primary $\mathrm{OA}$ & $\begin{array}{l}50 \% \text { decrease of medial joint } \\
\text { space }\end{array}$ & Clefts, softening & $5 \mathrm{yr}$ & 10 \\
\hline $\mathrm{F}$ & $59 \mathrm{M}$ & Primary OA & $\begin{array}{l}100 \% \text { decrease of medial } \\
\text { joint space }\end{array}$ & No data & $10 \mathrm{yr}$ & 100 \\
\hline G & $36 \mathrm{M}$ & $\mathbf{M M}$ & Normal & Normal & $1 \mathrm{wk}$ & 10 \\
\hline
\end{tabular}

* Trauma and rupture of anterior cruciate ligament (ACL), medial meniscus (MM), lateral meniscus (LM). Primary osteoarthritis with no history of knee joint injury (OA). ${ }^{\ddagger}$ Standing $x$-ray films. ${ }^{\S}$ At time of joint fluid sampling. "Time after injury or meniscectomy or duration of complaint. 'Volume of joint fluid used for isolation of aggrecan fragments. 

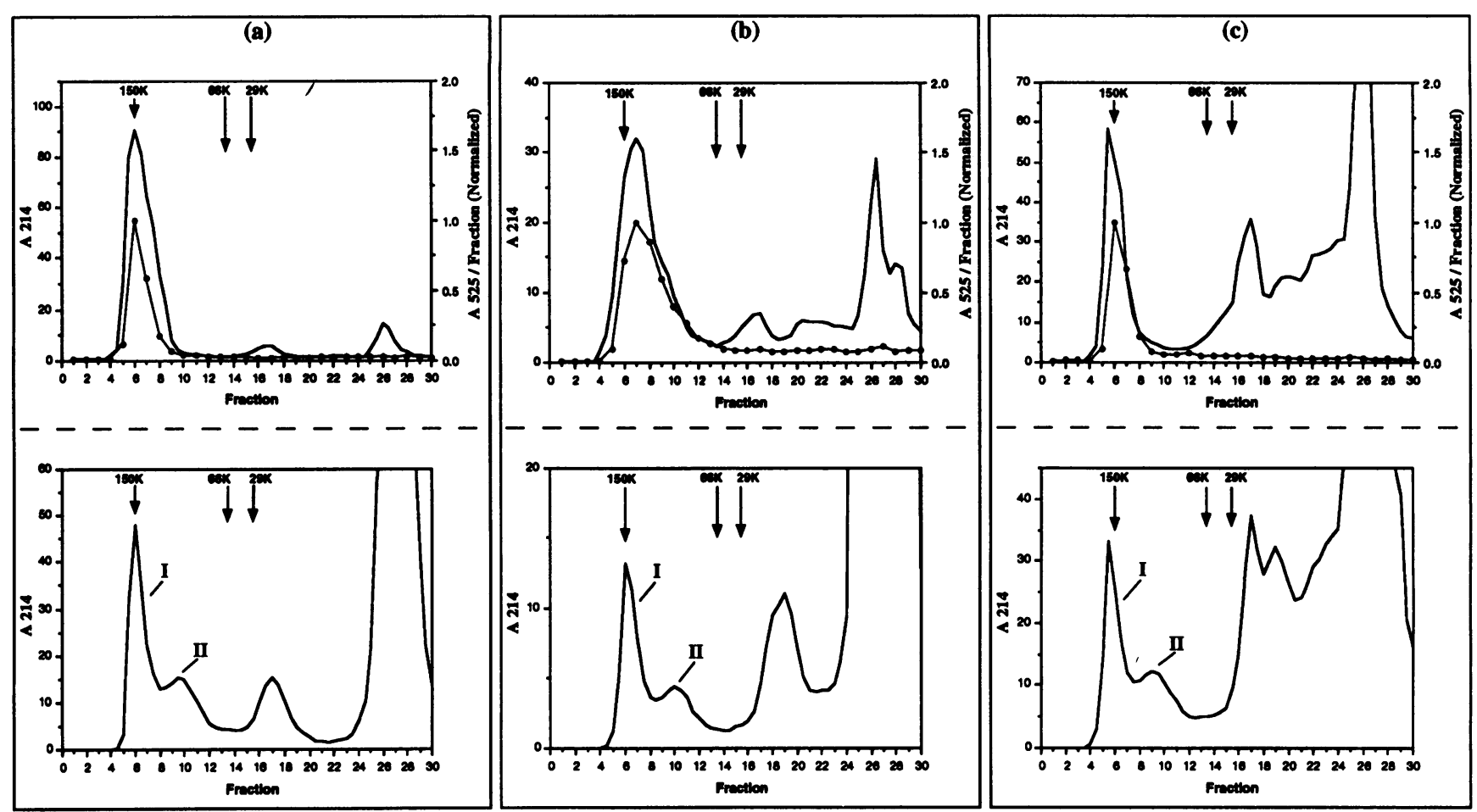

Figure 1. Superose-12 fractionation of aggrecan fragments from the synovial fluid of $(a)$ patient $\mathrm{A},(b)$ patient $\mathrm{D}$, and $(c)$ patient $\mathrm{F}$. (Upper panels) Aggrecan fragments (typically $400-600 \mu \mathrm{g}$ ) purified by $\mathrm{CsCl}$ gradient centrifugation from synovial fluids were applied to Superose-12 and eluted in $0.5 \mathrm{M}$ guanidine- $\mathrm{HCl}$ at $0.5 \mathrm{ml} / \mathrm{min}$ with collection of $0.5 \mathrm{ml}$ fractions. The eluant was monitored at $214 \mathrm{~nm}$ (continuous line) and fractions were assayed for CS (connected dots). (Lower panels) Deglycosylated aggrecan fragments (see Methods) were applied to Superose-12 as described above and the eluant was monitored at $214 \mathrm{~nm}$ (continuous line). Core protein species in pool I (fractions 4-8) and pool II (fractions 9-13) were taken for $\mathrm{NH}_{2}$-terminal sequencing. Molecular mass markers were carbonic anhydrase (29 kD), bovine serum albumin (66 kD), and alcohol dehydrogenase $(150 \mathrm{kD})$.

After deglycosylation, the $214 \mathrm{~nm}$ absorbance profiles for each sample (Fig. 1, lower panels) showed two major peaks between fractions 4 and 13 (labelled as I and II). The 214-nm absorbing species eluted between fractions 14 and 22 were apparently not generated by the deglycosylation step but were present in the starting material (note change in absorbance scale between upper and lower panels). The high absorbance at around fraction 26 corresponds to the column Vt and represents components and products of the deglycosylation procedure.

The proteins eluted in pools I and II (Fig. 1, lower panels) were both derived from the high molecular weight CS-bearing aggrecan fragments shown in the upper panels (fractions 4-10). Thus, when other portions of D1 samples from patients $A, D$, and $F$ were purified on Superose-12, and fractions 4-10 were pooled for deglycosylation, the 214-nm profile of these core samples on Superose-12 again showed the characteristic double peak in fractions 4-11. The D1 preparation from normal bovine synovial fluid showed essentially the same Superose-12 profiles as the human material, for both the untreated and the deglycosylated samples (profiles not shown).

$\mathrm{NH}_{2}$-terminal analysis of aggrecan core proteins from synovial fluids. The aggrecan core preparations from Superose-12 chromatography (pools I and II) of both human and bovine synovial fluid samples were taken for $\mathrm{NH}_{2}$-terminal analysis and the results, with approximate picomole yields, are shown in Table II.

For all synovial fluids analyzed, and for both pool I and pool II core protein species, the major sequence obtained (see for example, patient A Superose pool I, sequence ARGSVILXVKP) clearly corresponded to the human aggrecan sequence beginning ARGSVILTVKP, which initiates at Ala 374 within the interglobular domain (from the cDNA, ref. 17). Interestingly, the eighth residue could not be detected in any sample and since this is a threonine in the cDNA-derived sequence, it seems likely that this residue is modified, probably by $\mathrm{O}$-substituted carbohydrate. The finding of the same $\mathrm{NH}_{2}$ terminus on pool I and pool II core species suggests that their separation

Table II. $\mathrm{NH}_{2}-\mathrm{N}$ Terminal Sequence Data Obtained for DI Aggrecan Fragments Isolated from Synovial Fluids and Fractionated by Superose-12 Gel Filtration (Superose Pools Shown in Fig. 1, Lower Panels)

\begin{tabular}{lrlr}
\hline $\begin{array}{c}\text { Synovial } \\
\text { fluid }\end{array}$ & Superose pool & \multicolumn{1}{c}{ Sequence } & $\begin{array}{c}\text { Approximate } \\
\text { yield }\end{array}$ \\
\hline & & & $p m o l$ \\
Patient A & I & ARGSVILXVKP & 33 \\
& II & ARGSV & 32 \\
Patient D & I & AXGXVIL & 10 \\
& II & ARGSVILXVK & 39 \\
Patient F & I & ARGSV & 11 \\
& II & ARGSVILXVKPIFEVXP & 145 \\
Patient G & II & ARGSVIL & 11 \\
Bovine & I & ARGSVI & 13 \\
& II & AXGSVIL & 15 \\
\hline
\end{tabular}


by size is due to different $\mathrm{COOH}$ termini, consistent with limited cleavage within the CS domain(s) as discussed above.

\section{Discussion}

Large aggrecan fragments have previously been shown to be present in joint fluids from patients with inflammatory joint disease or OA (21). We now extend these findings and show that posttraumatic and osteoarthritic human synovial fluid contains at least two populations of aggrecan fragments, both of which are relatively large and both of which carry the $\mathrm{NH}_{2}$-terminal sequence ARGSVILXVK, situated within the interglobular domain. A diagrammatic representation of two possible structures is given in Fig. 2. It is clear that this $\mathrm{NH}_{2}$ terminus (Ala 374) was not generated by proteolysis during deglycosylation with chondroitinase and keratanase, nor at subsequent stages in the isolation of core proteins; thus in control studies with deglycosylated calf articular A1D1 aggrecan the only detectable $\mathrm{NH}_{2}$-terminal sequence obtained, VEVS, corresponded to that expected for the intact molecule (16). In addition, the $\mathrm{NH}_{2}$-terminal sequence FFGVGGEEDIXVQ (initiating at Phe 342) was obtained after deglycosylation of stromelysin-generated CS-bearing human aggrecan fragments, with no evidence for an $\mathrm{NH}_{2}$-terminal sequence beginning at Ala 374 (22).

Such species (Fig. 2) would appear to represent the bulk of the CS-bearing fragments present in osteoarthritic human synovial fluid for the following reasons. Firstly, greater than $94 \%$ of the fragments recovered from the $\mathrm{CsCl}$ gradient were recovered in the D1 fraction. Secondly, all of the fragments in such D1 fractions were present in the void volume on Superose-12 fractionation and deglycosylation of such samples generated only two populations of aggrecan core protein (termed pools I and II). Thirdly, the only detectable sequence in both pool I and pool II samples was ARGSVILXVK and the molar yield of this sequence was generally about $25 \%$ of the starting protein used in preparation of the samples (assuming a molecular mass of $150 \mathrm{kD}$ for these core proteins). This appears to be a reasonable yield, since two chromatographic steps, with attendant losses, were used in sample preparation for sequencing and the yield in Edman degradation is often only $40-80 \%$ of expected. It should be noted, however, that these analyses are confined to CS-bearing fragments of aggrecan and do not address the struc- ture or abundance of nonglycosylated fragments such as the G1 domain shown to be present in human synovial fluids by Witter and co-workers (21).

The results also show that essentially the same products are released into synovial fluid from normal mature bovine articular cartilage in vivo. In addition, our earlier studies (16) showed that the same $\mathrm{NH}_{2}$-terminal sequence was present on aggrecan fragments released from bovine cartilage in explant cultures both with and without IL-1 treatment. Similar studies on aggrecan fragments in the synovial fluid from rabbit joints injected with IL-1 have also shown the same major $\mathrm{NH}_{2}$-terminal sequence (McDonnell, J., V. Moore, R. Boynton, C. Flannery, P. Neame, and J. Sandy, unpublished observation).

When taken together, these studies indicate that the protease responsible for cleavage of the Glu 373-Ala 374 bond in the interglobular domain of aggrecan is widely distributed, and would appear to be part of a general mechanism for the catabolism of aggrecan in both normal turnover and in pathological situations. This implies that this enzyme is normally expressed by chondrocytes and that overexpression of this activity may be a key event in the pathogenesis of disease states involving accelerated cartilage degradation, such as osteoarthritis.

The finding of this predominant $\mathrm{NH}_{2}$-terminal sequence on aggrecan fragments in all of these situations does not imply that the enzyme which cleaves the Glu 373-Ala 374 bond is the only, or indeed primary enzyme involved in aggrecan catabolism. The results do suggest, however, that this enzyme acts on the majority of catabolized molecules and that it catalyzes the most $\mathrm{COOH}$-terminal cleavage within the interglobular domain. It is thus possible that this enzyme acts secondarily to other proteases which cleave the interglobular domain nearer to the $\mathrm{NH}_{2}$ terminus.

In this regard it may be relevant that stromelysin- 1 cleaves the interglobular domain of both human (22) and porcine aggrecan (23) at the Asn 341-Phe 342 bond. Further, a proportion of the G1 domain which accumulates in human articular cartilage has Asn 341 as a $\mathrm{COOH}$ terminus, indicative of stromelysin-1 action in situ; stromelysin-1 does not, however, appear to catalyze the cleavage of the Glu 373-Ala 374 bond (22).

The protease which cleaves the Glu 373-Ala 374 bond of aggrecan remains to be identified. It may be a member of the family of matrix metalloproteinases, many of which have now been shown to degrade aggrecan as effectively as stromelysin
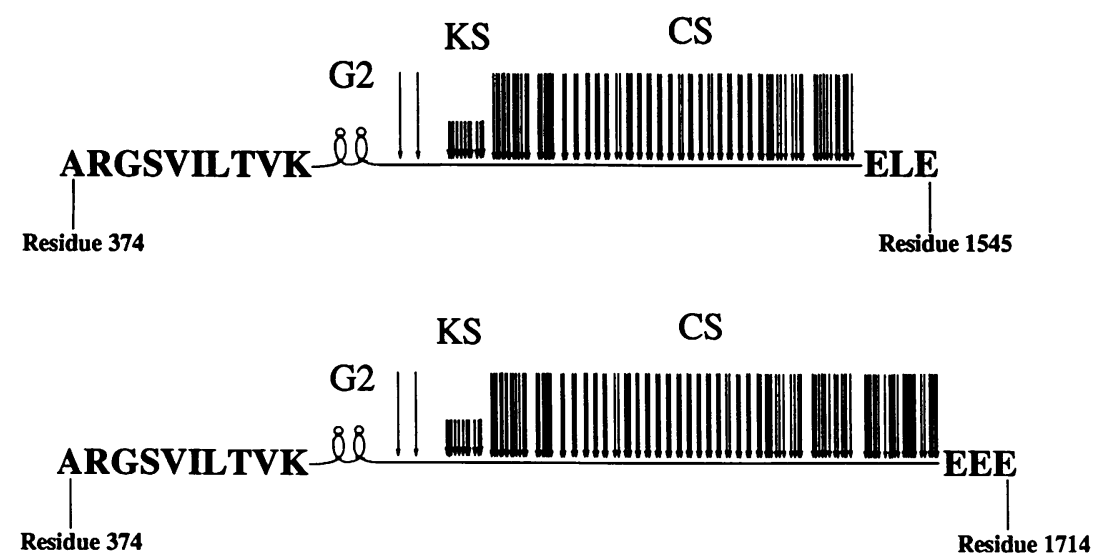

Figure 2. Diagrammatic representation of the possible structure of aggrecan fragments in synovial fluid. Two populations of aggrecan fragments are shown, both lacking the $\mathrm{G} 1$ domain, and both with the $\mathrm{NH}_{2}$-terminal sequence ARGSVILTVK adjacent to the $\mathrm{G} 2$ domain. The length of the CS attachment region on these species has not been directly determined but is predicted from the apparent size of the deglycosylated core proteins. The $\mathrm{COOH}$ termini as drawn also coincide with cleavage positions in the CS-domain described by Maniglia et al. (ref. 28) in bovine explant studies. Keratan sulfate $(K S)$ chains (small arrows) are shown only in the KS domain but some are also probably interspersed in the CS domain. CS chains (large arrows) are shown at all putative CS-attachment sites (Ser-Gly) predicted from the human sequence (ref. 17). 
(24). Alternatively, it might be a member of the cathepsin group of lysosomal cysteine proteinases (25). There is direct evidence that in human cartilage in vivo, a proportion of the link protein has been cleaved by cathepsin B which is presumably derived from the chondrocytes (26). Whatever the nature of this protease, it is almost certainly involved in generation of the short half-life pool of aggrecan seen in cartilage both in vivo and in vitro $(18,27)$. This would indicate that its activity might be particularly high in the pericellular matrix of cartilage where it would have access to newly secreted aggrecan, thereby implying that this enzyme plays a central role in the control of aggrecan deposition in cartilage matrix in both normal and diseased states.

The finding that products of this enzyme are present in the synovial fluid of patient A (3 days after anterior cruciate ligament rupture), patient $\mathrm{D}$ (15 years after medial meniscectomy), and patient $F$ ( 10 years of primary OA with extensive cartilage loss), suggests that expression of this activity may be both an early and persistent feature of the chondrocyte response to the joint changes associated with both primary and posttraumatic OA. Identification of the enzyme could allow a rational approach towards the development of a therapy with the aim of retarding or inhibiting cartilage matrix destruction in osteoarthritis.

\section{Acknowledgments}

The authors wish to acknowledge the excellent technical assistance of Raymond Boynton and Carmen Young.

This work was supported by a grant from the Shriners of North America, National Institutes of Health, grant AR 38580, the Swedish Medical Research Council (8713), the King Gustaf V 80-year fund, the Ax:son Johnson and Kock Foundations and the Medical Faculty of Lund Univeristy.

\section{References}

1. Hoch, D. H., A. J. Grodzinsky, T. J. Koob, M. L. Albert, and D. R. Eyre. 1983. Early changes in material properties of rabbit articular cartilage after menisectomy. J. Orthop. Res. 1:4-12.

2. Carney, S. L., M. E. J. Billingham, H. Muir, and J. D. Sandy. 1984. Demonstration of increased proteoglycan turnover in cartilage explants from dogs with experimental osteoarthritis. J. Orthop. Res. 2:201-206.

3. Carney, S. L., M. E. J. Billingham, H. Muir, and J. D. Sandy. 1985. Structure of newly synthesized (35S)-proteoglycans and (35S)-proteoglycan turnover products of cartilage explant cultures from dogs with experimental osteoarthritis. J. Orthop. Res. 3:140-147.

4. Lohmander, L. S., L. Dahlberg, L. Ryd, and D. Heinegård. 1989. Increased levels of proteoglycan fragments in knee joint fluid after injury. Arthritis Rheum. 32:1434-1442.

5. Lohmander, L. S., L. Dahlberg, L. Ryd, and D. Heinegård. 1990. Joint markers in synovial fluid in human osteoarthritis. Trans. Orthop. Res. Soc. 15:212. (Abstr.)

6. Lohmander, L. S., and L. Dahlberg. 1991. Proteoglycan epitopes in joint fluid in human osteoarthritis. Trans. Orthop. Res. Soc. 16:227. (Abstr.)
7. Docherty, A. J. P., and G. Murphy. 1990. The tissue metalloproteinase family and the inhibitor TIMP: a study using CDNAs and recombinant proteins. Ann. Rheum. Dis. 49:469-479.

8. Murphy, G., R. M. Hembry, C. E. Hughes, A. J. Fosang, and T. E. Hardingham. 1990. Role and regulation of metalloproteinases in connective tissue turnover. Biochem. Soc. Trans. 18:812-815.

9. Dean, D. D., J. Martel-Pelletier, J.-P. Pelletier, D. S. Howell, and J. F. J. Woessner. 1989. Evidence for metalloproteinase and metalloproteinase inhibitor imbalance in human osteoarthritic cartilage. J. Clin. Invest. 84:678-685.

10. Lohmander, L. S., L. A. Walakovits, and M. W. Lark. 1992. Metalloproteinases, tissue inhibitor and proteoglycan fragments in knee synovial fluid in human osteoarthritis. Trans. Orthop. Res. Soc. 17:273. (Abstr.)

11. Lohmander, L. S., L. A. Walakovits, L. Dahlberg, H. Roos, and M. W. Lark. 1992. Temporal patterns of release of stromelysin, tissue inhibitor and proteoglycan fragments in synovial fluid after knee injury. Trans. Orthop. Res. Soc. 17:651. (Abstr.)

12. MacNaul, K. L., N. Chartrain, M. Lark, M. J. Tocci, and N. I. Hutchinson. 1990. Discoordinate expression of stromelysin, collagenase, and tissue inhibitor of metalloproteinases- 1 in rheumatoid synovial fibroblasts. J. Biol. Chem. 265:17238-17245.

13. Gravallese, E. M., J. A. Darling, A. L. Ladd, J. N. Katz, and L. H. Glimcher. 1991. In situ hybridization studies of stromelysin and collagenase messenger RNA expression in rheumatoid synovium. Arthritis Rheum. 34:10761084.

14. McCachren, S. S. 1991. Expression of metalloproteinases and metalloproteinase inhibitor in human arthritic synovium. Arthritis Rheum. 34:1085-1093.

15. Firestein, G. S., M. M. Paine, and B. H. Littman. 1991. Gene expression (collagenase, tissue inhibitor of metalloproteinases, complement and HLA-DR) in rheumatoid arthritis and osteoarthritis synovium. Arthritis Rheum. 34:10941105.

16. Sandy, J. D., P. J. Neame, R. E. Boynton, and C. R. Flannery. 1991. Catabolism of aggrecan in cartilage explants. Identification of a major cleavage site within the interglobular domain. J. Biol. Chem. 266:8683-8695.

17. Doege, K., M. Sasaki, T. Kimura, and Y. Yamada. 1991. Complete coding sequence and deduced primary structure of the human cartilage large aggregating proteoglycan, aggrecan. J. Biol. Chem. 266:894-902.

18. Sandy, J. D. 1992. Extracellular metabolism of aggrecan. In Articular Cartilage and Osteoarthritis. K. Kuettner, et al., editors. Raven Press, Ltd., New York. 21-33.

19. Lohmander, L. S. 1991. Markers of cartilage matrix metabolism in arthrosis. A review. Acta Orthop. Scand. 62:623-632.

20. Farndale, R. W., C. A. Sayers, and A. J. Barrett. 1982. A direct spectrophotometric microassay for sulfated glycosaminoglycan in cartilage cultures. Connect. Tissue Res. 98:247-248.

21. Witter, J., P. J. Roughley, C. Webber, R. Roberts, E. Keystone, and A. R Poole. 1987. The immunologic detection and characterization of cartilage proteoglycan degradation products in synovial fluids of patients with arthritis. Arthritis Rheum. 30:519-526.

22. Flannery, C. R., M. W. Lark, and J. D. Sandy. 1992. Identification of a stromelysin cleavage site within the interglobular domain of human aggrecan: evidence for proteolysis at this site in vivo in human articular cartilage. J. Biol. Chem. 267:1008-1014.

23. Fosang, A. J., P. J. Neame, T. E. Hardingham, G. Murphy, and J. A Hamilton. 1991. Cleavage of cartilage proteoglycan between $G 1$ and G2 domains by stromelysins. J. Biol. Chem. 266:15579-15582.

24. Murphy, G., M. I. Cockett, R. V. Ward, and A. J. Docherty. 1991. Matrix metalloproteinase degradation of elastin, type IV collagen and proteoglycan. Biochem. J. 277:277-279.

25. Barrett, A. J., D. J. Buttle, and R. W. Mason. 1988. Lysosomal cysteine proteinases. ISI Atlas of Science Biochem. 256-260.

26. Nguyen, Q., J. Liu, P. J. Roughley, and J. S. Mort. 1991. Link protein as a monitor in situ of endogenous proteolysis in adult human articular cartilage. Biochem. J. 278:143-147.

27. Lohmander, L. S. 1977. Turnover of proteoglycans in guinea pig costal cartilage. Arch. Biochem. Biophys. 180:93-101.

28. Maniglia, C. A., P. P. Loulakis, A. V. Shrikhande, and G. Davis. 1991 IL-1 elevated PG degradation reveals $\mathrm{NH}_{2}$-terminal sequence homology. Trans. Orthop. Res. Soc. 16:193. (Abstr.) 